\title{
Le Master Migrations internationales, espaces et sociétés à Poitiers
}

Fondements théoriques, évolution de l'offre de formation et perspectives d'internationalisation

David Lessault, Adelina Miranda et Véronique Lassailly-Jacob

\section{(2) OpenEdition}

\section{Journals}

Édition électronique

URL : https://journals.openedition.org/e-migrinter/844

DOI : $10.4000 /$ e-migrinter.844

ISSN : 1961-9685

Éditeur

UMR 7301 - Migrinter

Référence électronique

David Lessault, Adelina Miranda et Véronique Lassailly-Jacob, "Le Master Migrations internationales, espaces et sociétés à Poitiers », e-Migrinter [En ligne], 15 | 2017, mis en ligne le, consulté le 20 mai 2021. URL : http://journals.openedition.org/e-migrinter/844 ; DOI : https://doi.org/10.4000/e-migrinter. 844

Ce document a été généré automatiquement le 20 mai 2021.

Tous droits réservés 


\section{Le Master Migrations internationales, espaces et sociétés à Poitiers}

Fondements théoriques, évolution de l'offre de formation et perspectives d'internationalisation

David Lessault, Adelina Miranda et Véronique Lassailly-Jacob

1 Migrinter est un lieu de recherche et de formation. ${ }^{1}$ C'est pourquoi il a été jugé utile de dédier un temps du colloque anniversaire des 30 ans de Migrinter à une discussion collective sur l'enseignement et la formation. Le panel « Comment enseigne-t-on les migrations internationales dans les Sciences Humaines et Sociales?» a traité plus particulièrement de l'enseignement dispensé dans les Masters spécialisés dans le domaine des «migrations internationales ». En France, ces derniers sont encore rares. S'il existe des modules "migrations » ou " mobilités » internationales dans différents Masters en Sciences Humaines et Sociales (SHS), peu d'entre eux s'inscrivent dans une mention ou spécialité orientée sur l'objet même.

2 En effet, l'étude des migrations internationales ne s'est structurée en France qu'autour de quatre acteurs académiques: l'Unité Mixte de Recherche (UMR) Migrinter (Université de Poitiers/CNRS), l'UMR URMIS (Universités Paris VII et Nice SophiaAntipolis, CNRS, IRD), le Centre d'Études sur les Relations Internationales (Sciences Po Paris) et l'Unité de Recherche (UR) «Migrations internationales et minorités » (Institut National d'Études Démographiques). Au sein de ce paysage national, deux Masters seulement, celui adossé à Migrinter « Migrations internationales, espaces et sociétés " et celui adossé à l'URMIS «Migrations et relations interethniques» ont dans leur intitulé le terme de «Migrations ». La dynamique scientifique du laboratoire Migrinter s'inscrit dans une articulation entre la géographie (discipline d'origine) et d'autres disciplines auxquelles l'équipe s'est ouverte : droit, histoire, socio-anthropologie. Cette dynamique confère à la formation sa singularité dans le paysage universitaire national, voire international, appréhendant les différentes facettes de la migration au prisme d'une spatialisation de la réflexion. 
3 Le Master «Migrations internationales, espaces et sociétés » est, depuis ses origines, adossé à Migrinter (CNRS/Université de Poitiers) et au Département de Géographie. Le contenu de la formation et les objets de recherche s'inscrivent dans les axes de recherche de l'UMR. La majorité des compétences scientifiques en sont issues. La formation est destinée à de futurs doctorants, mais également à des étudiants qui ne poursuivront pas une formation doctorale. La recherche d'emploi dès la sortie du Master constitue une pratique fréquente pour les étudiants et elle est prise en compte dans l'organisation des enseignements et dans les dispositifs pédagogiques mis en œuvre tout au long de leur formation. La structure et l'évolution des cours dispensés sont le reflet d'une pratique du terrain confirmée chez les chercheurs du laboratoire (géographes, historiens, sociologues, anthropologues, juristes) et relève donc d'une diversité de compétences et d'approches selon les disciplines. À cette première source de pluralité pédagogique s'ajoute celle des étudiants qui intègrent la formation. Ces derniers sont d'origines disciplinaires et nationales différentes. Ce recrutement, source de parcours et d'intentions professionnelles variés, traduit le rayonnement international $\mathrm{du}$ Master. Il conduit également à une adaptation des méthodes d'enseignement et à une réflexion sur les réponses à apporter sur l'internationalisation du public attiré par l'étude des migrations internationales.

4 Cet article est une synthèse des communications proposées au colloque de l'UMR Migrinter $^{2}$ par Véronique Lassailly-Jacob sur les origines et l'histoire du Master à Poitiers (France), Pierre Kamdem sur la création d'un Master professionnel sur les relations « Migrations et développement » à Dschang (Cameroun) et Papa Sakho sur le développement du Master «Migrations Internationales, Développement et Relations Interculturelles » (MIRI) de Dakar (Sénégal). Ces trois interventions, complétées par les réflexions de David Lessault, responsable du Master "Migrations internationales, espaces et sociétés» (Poitiers) de 2013 à 2016 et Adelina Miranda, actuelle coresponsable avec Christophe Imbert, structurent le propos. Les auteurs soulignent les fondements théoriques et les grandes évolutions pédagogiques du Master migrations internationales de Poitiers et soulèvent finalement le défi et les difficultés actuelles de son internationalisation, notamment par le biais de coopérations universitaires avec des partenaires africains. Cette synthèse contribue également à alimenter la discussion critique lancée par Swanie Potot dans sa communication présentée au colloque de l'UMR Migrintersur le Master de l'URMIS à Nice: "comment dépasser un certain nationalisme méthodologique et penser un système d'enseignement international sur les migrations?». Enfin, elle souligne l'influence déterminante des contextes scientifiques et politico-institutionnels qui se traduit par des ruptures épistémologiques dans le déroulement des formations mises en place. L'article est agrémenté par les témoignages d'anciens étudiants ayant suivi la formation à Poitiers.

\section{Le Master « Migrations internationales espaces et sociétés ", historiquement adossé au laboratoire Migrinter}

5 On ne peut comprendre l'évolution du Master « Migrations Internationales espaces et sociétés » sans évoquer l'histoire du laboratoire Migrinter et y associer celle de son fondateur, Gildas Simon. 
6 En 1985, Gildas Simon, professeur de géographie à l'Université de Poitiers, obtient auprès du CNRS la création d'un laboratoire centré sur l'étude des migrations internationales de travail et leurs liens avec les sociétés d'origine (Unité de Recherche Associée 1145).

7 C'est le premier centre de recherche créé par le CNRS en géographie des migrations internationales. En effet, jusque dans les années 1970-1980, les recherches sur les migrations et les relations interethniques sont encore peu développées en France ; à la différence des pays anglo-saxons, notamment les États-Unis où les travaux fondateurs de recherches en sociologie urbaine de la première "École de Chicago » entre 1915 et 1940 se sont prolongés par une seconde «École de Chicago » (1964-1973), davantage fondée sur des sociologies interactionnistes et empiriques (Coulon, 2007). À cette époque, en France, les migrations sont plutôt étudiées par les démographes et par une certaine "géographie de la population» (Mauco, 1932). Elles commencent à peine à être considérées comme un objet de recherche scientifique au sein de la géographie française (Noin, 1979).

8 Les premiers travaux sont consacrés aux migrations de travail et à l'impact des transferts sur les sociétés d'origine, thèmes fondateurs des recherches du laboratoire. Une attention particulière est accordée aux migrations de travail des pays du Maghreb. Les effets de la migration y sont étudiés à travers la mobilisation de nouveaux concepts géographiques appliqués aux migrations internationales tels que les espaces ou les champs migratoires, les filières et réseaux, les circulations et les diasporas ouvrières.

Les fondements théoriques et méthodologiques de l'époque s'appuient largement sur les notions d'espace et de champ migratoire dont la définition a permis de mettre en perspective une dimension spatiale des phénomènes migratoires, peu abordée par les disciplines connexes des sciences sociales. Les textes de Gildas Simon (1979) et Roger Béteille (1981) sur la notion de champ migratoire sont devenus des références incontournables, mobilisées pour enseigner les migrations dans une perspective géographique. En effet, le champ migratoire apparaît comme une notion charnière de la recherche sur les migrations internationales à Poitiers puisqu'il précise une position scientifique par rapport au concept plus neutre de système migratoire utilisé dans d'autres disciplines des SHS ; cette notion s'est progressivement affirmée comme le support théorique du prolongement des réflexions autour de la notion de circulation migratoire. Dès lors, les chercheurs se sont projetés sur des terrains situés aussi bien au Sud, dans les pays de départ, qu'au Nord, dans les pays d'arrivée.

10 En 1986, le laboratoire prend le nom de Migrinter, nouveau pôle scientifique associant des activités de recherche, de formation, de publication et de documentation sur les migrations internationales, notamment sur les reconfigurations sociales et spatiales qu'elles génèrent. Le master de Migrinter reflète l'étendue de son réseau national et international, et souligne une ouverture disciplinaire assez précoce: il a presque d'emblée une assise "transnationale ». Son fonctionnement s'affranchit rapidement des contraintes frontalières sous l'effet des pratiques scientifiques de ses enseignants fortement ancrés sur des terrains à l'étranger mais également sur la diversité et la dispersion internationale des terrains investis par les étudiants. 


\section{Le Diplôme d'Études Approfondies (DEA) « Migrations, Espaces, Sociétés » : une formation multi-sites et pluridisciplinaire (1991-2005)}

11 La création en 1991 du DEA « Migrations, Espaces, Sociétés » constitue un temps fort de l'histoire du laboratoire. Deux ans plus tard, la formation est co-habilitée avec l'URMIS, (Unité mixte de Recherche Migrations et Sociétés sous la tutelle des universités Paris VII Denis Diderot et Nice Sophia-Antipolis). Le DEA, devenu multi-sites, renforce sa dimension pluridisciplinaire (géographie, sociologie, anthropologie), s'ouvre à l'étude des relations interethniques ce qui le conduit à changer son intitulé : "Migrations et relations interethniques ». Chaque site privilégie l'enseignement des objets et méthodes propres à sa discipline. Le site de Poitiers aborde alors plus spécifiquement la problématique des migrations internationales dans sa dimension spatiale. L'approche théorique d'ensemble se concentre sur quatre thèmes : le migrant acteur de sa mobilité géographique et sociale, les espaces matériels et symboliques de la migration, les minorités et les identités collectives et enfin, les politiques migratoires et d'asile. Quant aux quatre unités d'enseignement du tronc commun enseigné dans les trois sites, elles s'intitulent "Penser l'étranger: lecture et discussion de textes fondamentaux", «Vocabulaire des migrations et des relations interethniques », " Espace et mobilité » et «Mise en œuvre des méthodes aux spécificités du domaine ». Les étudiants suivent les enseignements du tronc commun de leur site ainsi que les cours disciplinaires ou les enseignements de spécialité offerts par les deux autres sites. Cette circulation étudiante, enrichissante et stimulante, dût s'arrêter en 2004 en raison des difficultés de gestion administrative de cette formation multi-sites.

Durant cette période, les enseignements à Poitiers traitent des circulations migratoires et du transnationalisme, des mobilités et de la production de l'urbain. D'une manière plus spécifique la notion de circulation migratoire sera partagée par l'ensemble des disciplines de Sciences Humaines et Sociales investies dans la description de la mondialisation. Cette notion peut «recouvrir des situations migratoires assez distinctes. Mais on peut s'accorder pour dire que prédomine toujours l'idée d'une mobilité comme un élément organisateur fort des dynamiques sociales pour des individus et des groupes ayant été placés en situation migratoire. (...) Dans ce sens, le terme renvoie aux mobilités des individus, mais aussi des biens et des valeurs, dans un espace structuré antérieurement par des flux migratoires, c'est-à-dire dans un « champ migratoire » (Simon, 1979) (et où) la notion de circulation vise à ne restreindre l'analyse de la migration ni aux seules problématiques du départ ou de l'installation, ni à les placer dans des temporalités dont seraient exclues la persistance et la réversibilité des mouvements (Domenach, Picouet, 1987). Que ce soient des parcours de migration circulaire avec retour au point de départ ou des mobilités observables dans les deux sens, « c'est la mobilité alternante, à caractère répétitif et cyclique, entre un pays et un autre, reliant une ville à une autre, un village à une métropole, souvent sous le mode du «va-et-vient » entre deux ou plusieurs lieux et échappant par la même aux définitions traditionnelles de la migration qui semble pouvoir constituer un fil conducteur de l'approche des migrations par la circulation » (Cortès et Faret, 2009, p. 12).

13 À côté d'une géographie des migrations internationales qui se pérennise par la présence marquée de géographes au sein du laboratoire et leur approche en termes de circulation, la sociologie et l'anthropologie continuent d'occuper une place importante 
au sein des enseignements. La sociologie des migrations est développée à partir de la problématique de l'étranger dans la ville et s'appuie sur une lecture critique des travaux de «l'École de Chicago » (Missaoui, Tripier, Hily, 2002). La mise en scène de l'étranger dans la ville révèle en même temps l'existence des formes d'interaction et des frontières entre insertion et exclusion, entre identité et altérité. La sociologie des migrations privilégie aussi l'étude des réseaux sociaux en migration. Au milieu du $\mathrm{XX}^{\text {ème }}$ siècle, de nouvelles formes migratoires se sont juxtaposées aux migrations de travail règlementées par les États européens. Une approche socio-anthropologique de l'économie souterraine transnationale autour du bassin méditerranéen a révélé l'existence des "fourmis» de la mondialisation, nomades invisibles des temps modernes (Tarrius, 1992). Ces populations circulantes, soudées par un solide réseau souterrain, produisent des territoires particuliers transcendant les États-nations. L'approche de cette circulation redéfinit les concepts de migrant, de nomade et d'errance (Tarrius, 2002). L'économie des migrations est également abordée dans les pays d'origine sous l'angle des transferts de biens et d'argent des migrants, nouvelle manne convoitée par les États dans le cadre de leurs stratégies de développement. Dans les pays d'accueil, de nombreux mémoires d'étudiants travaillent la question du « commerce ethnique » impulsée par les recherches d'Emmanuel Ma Mung notamment (Ma Mung, 1992).

\section{Adaptations et évolutions pédagogiques du Master «Migrations Internationales » (2005-2016)}

Dans le cadre de la mise en place du «LMD » (Licence-Master-Doctorat), le DEA devient en 2005 un Master recherche mono-site et pluridisciplinaire, intitulé "Migrations internationales et relations interethniques"(MIRI). L'année suivante, est créé le Master professionnel «Migrations Internationales, Conception de projets en coopération pour le développement » (MICP) aux côtés du Master recherche rebaptisé «Migrations internationales, Espaces et Sociétés » (MIES). Cette nouvelle architecture s'accompagne d'un remaniement de l'équipe pédagogique qui ouvre de nouvelles voies à la formation en migrations internationales.

Les diplômés du Master...

Après ma licence de géographie, j'ai choisi d'intégrer le Master « Migrations internationales, Espaces et Sociétés ". C'est une formation qui me permettait à la fois de me spécialiser dans le domaine des mobilités de population et des migrations internationales et de travailler spécifiquement sur un thème qui m'était cher et qui m'intriguait : la vie des Gens du Voyage en France. La première année du Master, en m'initiant aux premiers rudiments d'une démarche de recherche, m'a permis de mieux comprendre la nature des débats publics et politiques souvent passionnés sur la présence des Gens du Voyage en France. Prenant conscience que le " problème » reposait finalement sur la méconnaissance des groupes sociaux que j'avais observés, j'ai décidé d'aller plus au fond des choses en suivant l'année suivante les enseignements du Master Recherche. Les cours théoriques et les techniques d'enquête qui m'ont été transmises ont complètement changé ma vision du sujet. J'ai découvert le " gouffre " qui existait entre la vision caricaturale des Gens du 
Voyage véhiculée dans les sphères médiatiques et politiques et les résultats d'une observation menée en toute neutralité avec une rigueur scientifique.

Encouragée par l'équipe pédagogique du Master, j'ai souhaité défendre ma position et mon approche de la question en m'engageant dans une thèse de Doctorat. N'ayant pas obtenu de bourse d'allocation, j'ai réalisé ma thèse en alternant plusieurs activités professionnelles en qualité d'Ingénieur d'Études sur des programmes de recherche du CNRS et de l'IRD. Si le Master Recherche donne accès à la thèse, il transmet aussi des compétences spécifiques (Bac+5) qui permettent d'occuper des postes très intéressants: ces expériences successives ont certainement consolidé mon savoir-faire et m'ont ouvert de nouvelles portes.

J'ai ainsi pu être recrutée comme Attaché Temporaire d'Enseignement et de Recherche (ATER) à l'Université de Poitiers. Enseigner pendant la réalisation de ma thèse ne fut pas chose aisée, mais c'est ce qui m'a conforté dans mon choix de devenir enseignantchercheur. Dans ma discipline, la Géographie, de nouveaux outils informatiques ont radicalement changé les méthodes de travail. L'Université de Poitiers offre, à côté du Master, une formation en Système d'Information Géographique (SIG) et en analyse statistique et spatiale (Diplôme Universitaire SIGmage). J'ai bénéficié de cette formation complémentaire pour m'adapter aux nouvelles techniques d'analyse et être autonome dans mon métier de chercheur et d'enseignant.

Ayant soutenu ma thèse et mon mémoire du Diplôme Universitaire (DU) en 2011, j'ai été recrutée dès le lendemain à Paris par l'Organisation de Coopération et de Développement Économiques (OCDE) sur un poste de cartographe. L'année suivante, j'ai eu un poste d'ATER à temps complet à l'Université de Limoges. Les compétences progressivement acquises depuis mon Master Recherche, m'ont permis de répondre à une diversité d'offres d'emploi (chargée de mission dans les collectivités territoriales, concours de chercheur et d'enseignant chercheur, concours d'ingénieure de recherche, cartographe, etc.) et d'être opérationnelle sur différents types de poste.

Céline BERGEON, Maître de Conférences en Géographie à l'Université de Poitiers.

D'un côté, l'arrivée de nouveaux enseignants enrichit et élargit le socle commun des enseignements basé jusqu'alors sur les concepts fondateurs évoqués (champ et circulation migratoire; transnationalisme) et leur application empirique à différents groupes et dans diverses régions $\mathrm{du}$ monde. Certes l'approche géographique et systémique des migrations prédomine encore sous la forme de la production d'atlas (Robin, 1996; Clochard, 2012), mais c'est bien l'élargissement du spectre des déplacements à de nouvelles formes de mobilité qui marque l'évolution de la formation à partir de 2005. Par exemple, une nouvelle unité d'enseignement traite des crises et migrations forcées en analysant à la fois le rôle des États et des organisations humanitaires dans l'assistance et la protection des réfugiés ainsi que les parcours des réfugiés et leur articulation à plusieurs espaces ( Doraï, 2006; Lassailly-Jacob, 2003). Avec l'émergence d'une lecture multi-scalaire des mobilités spatiales (Chardonnel et al., 2009 ) et non plus uniquement des migrations internationales, l'appareillage méthodologique et théorique s'enrichit d'autres références, de nouveaux outils statistiques et cartographiques qui donnent une coloration plus " démo-géographique " et urbaine au Master: les enseignements méthodologiques orientés vers l'approche quantitative et la création d'une Unité d'Évaluation (UE) spécialement dédiée aux 
questions urbaines marquent cette transformation. Dans cette perspective, l'approche biographique des dynamiques spatiales permet à la fois une meilleure prise en compte du temps dans l'étude des migrations et une lecture éclairée des articulations diverses entre les pratiques individuelles et les mutations territoriales (Dureau, 1999; Imbert et al., 2014). L'unité d'enseignement «Lire les échelles de la mobilité » constitue un outil pédagogique permettant de situer les migrations internationales dans l'ensemble des pratiques de déplacement et de dépasser le cadre théorique des études migratoires pour se confronter et dialoguer avec d'autres champs scientifiques (Dureau, Hily, 2009). Dans ce dialogue interdisciplinaire, la place nouvelle de l'histoire, de l'anthropologie puis du droit s'affirme avec le recrutement de chercheurs spécialistes de ces disciplines et qui vont s'impliquer dans la formation.

De l'autre côté, l'ouverture de la voie professionnelle du Master "Migrations Internationales" dégage de nouvelles perspectives. La relation migrationdéveloppement, alors objet de controverses scientifiques (Guengant, 1996; Lacroix, Gonin, 2005), constitue l'un des axes de réflexion du projet de laboratoire au tournant des années 2000. Considérer le migrant comme acteur potentiel du développement de son pays d'origine devient la clé d'entrée de cette seconde voie du Master. En effet, la mise en relation des territoires par la migration appelle une déclinaison de la problématique du développement dans certains pays du sud qui constituent d'importants bassins d'émission des migrations internationales vers les pays de l'Organisation de Coopération et de Développement Économiques (OCDE). Dans ce contexte, la démarche pédagogique adoptée vise à associer aux enseignements fondamentaux dispensés par les chercheurs du laboratoire des interventions de professionnels investis sur le terrain dans des actions à destination des migrants ou de leur pays d'origine (Organisation non-gouvernementale, collectivités territoriales, travailleurs sociaux, etc.).

D'une manière générale, l'actualité de la dernière décennie a contribué à stimuler le besoin de connaissances sur les questions migratoires, d'autant plus qu'elles sont désormais liées à d'autres préoccupations sociétales majeures : changement climatique, liberté de circulation, droit des personnes, etc. Certaines catégories de migrants comme les " mineurs isolés étrangers ", les "réfugiés climatiques ", les "demandeurs d'asile » et les «femmes » font l'objet d'un intérêt croissant ; l'équipe pédagogique s'est adaptée en proposant de nouveaux séminaires dédiés à ces questions. Cependant, dans tous les cas, c'est la diversité des terrains, des approches et des compétences de l'équipe pédagogique qui permet de prendre un recul critique sur la fabrique des catégories sociales, politiques, juridiques, statistiques et sur la relativité des phénomènes migratoires d'actualité qui sont systématiquement replacés dans le temps long de l'histoire et dans l'ensemble des formes de déplacements humains. Aussi, qu'il s'agisse de la voie recherche ou professionnelle, les étudiants du Master sont formés dès leur première année à une pratique spécifique du terrain qui marque le caractère de la formation; une certaine "culture du terrain» qui est intimement articulée aux compétences et aux réseaux des chercheurs du laboratoire.

\section{Les diplômés du Master...}

Une fois diplômée d'une licence de géographie à Bordeaux 3, je souhaitais poursuivre mes études dans un cursus ayant une dimension internationale. En parcourant les offres de 
formation j'ai pris connaissance du Master « Migrations internationales, Espaces et Sociétés » proposé à Poitiers.

La première année de master a été l'occasion de poser les bases d'un champ pluridisciplinaire passionnant grâce aux enseignements délivrés par des géographes, des sociologues, des historiens, des démographes, etc.; mais aussi de faire la rencontre d'une promotion composée de personnes venues du monde entier et issues de disciplines très diverses (science politique, littérature, histoire, science de l'éducation etc.). Cette première année a également été celle d'une initiation à la recherche : construction d'un sujet, réalisation de recherches bibliographiques, pratique du terrain, restitution écrite et orale d'un mémoire.

Arrivée en Master avec l'optique d'intégrer sa spécialisation professionnelle l'année suivante, cette première année m'a fait prendre conscience de mon intérêt pour la recherche. En Master 2 (spécialité recherche) les enseignements reçus articulés à un temps entièrement consacré à la réalisation d'une deuxième recherche ont renforcé mon attrait pour le domaine de la recherche scientifique. Mais au-delà de ces considérations, c'est bien l'expérience humaine vécue à la fois avec mes collègues de promotion, l'équipe enseignante et surtout celle de mon terrain d'étude dans un foyer d'accueil pour mineurs isolés étrangers que je retiens aujourd'hui. Il ne faut pas s'y tromper, ce Master demande un investissement certain, d'ailleurs, dans les moments de doutes que notre promotion a pu rencontrer, l'équipe pédagogique a toujours su trouver les bons mots pour nous remobiliser.

Forte des enseignements reçus, des rencontres amicales ou professionnelles, $d u$ cheminement personnel parcouru,j'ai décidé de postuler à un poste de thèse. Soutenue par des membres de l'équipe enseignante dans cette nouvelle aventure, je réalise actuellement ma deuxième année de thèse. Dans le bureau des doctorants, ce sont certains de mes anciens camarades de Master que je retrouve avec le plus grand plaisir dans le couloir, mes anciens professeurs devenus des collègues de travail, mes tuteurs qui m'avaient suivi pendant mes deux années de Master sont aujourd'hui mes directeurs de thèse.

Sarah PRZYBYL, Docteure en Géographie de l'Université de Poitiers.

Dans l'objectif de favoriser la mise en place d'une formation à la recherche par la recherche, l'offre d'enseignements tire ses ressources dans les compétences pédagogiques et scientifiques du laboratoire. Le travail de concert entre universitaires et chercheurs des Établissements Publics Scientifiques et Techniques (EPST comme le CNRS et l'IRD) au sein de la formation s'incarne dans le fait que les différentes composantes du Master 1 (M1) et Master 2 (M2) ont chacune, depuis la création du Master, été gérées par un binôme associant un enseignant-chercheur et un chercheur du laboratoire. Plus de la moitié des enseignements du Master sont assurés, depuis plusieurs années, par des chercheurs, ingénieurs et techniciens des EPST membres du laboratoire ou associés. Le contenu pédagogique laisse une grande place aux recherches en cours et les étudiants du Master découvrent les projets structurants du laboratoire. Les chercheurs des EPST s'impliquent par ailleurs dans le suivi des mémoires des étudiants et dans les séminaires de formation par la recherche. Les domaines de recherche $\mathrm{du}$ laboratoire sont ainsi progressivement intégrés aux unités d'enseignement au fil de ses évolutions et du renouvellement de l'équipe scientifique. Dans la spécialité «Recherche » les unités d'enseignement se fondent sur les grands 
axes de recherche collectifs du laboratoire: systèmes migratoires et dynamiques spatiales; construction de l'altérité, politiques publiques et discriminations; méthodologie de la recherche sur les migrations internationales; construction régionale et politiques migratoires ; dynamiques urbaines et mobilités.

Cette articulation étroite entre la dynamique scientifique du laboratoire et le Master explique la variété de l'origine géographique et disciplinaire des étudiants recrutés. A l'image du rayonnement de l'UMR et des réseaux dans lesquels elle est insérée, le recrutement des étudiants est marqué par une grande ouverture pluridisciplinaire et une forte attractivité au-delà de Poitiers et à l'international. Trois grands types de parcours conduisant au Master 2 Recherche peuvent être distingués: les "locaux» ayant été formé en Licence à l'Université de Poitiers et ayant intégré le M1 Migrations internationales avant d'entrer en M2 dans la voie "Recherche»; les "nationaux" ayant été formés dans une autre université française et qui sont passés ou non par le M1; les « internationaux », étudiants venus de l'étranger pour intégrer la formation via le M1 ou directement en M2R. Le résultat montre clairement que le M2R est alimenté depuis «l'extérieur » avec de préférence un passage par le M1 mais pas toujours. Le bassin de recrutement du M2R est extra-régional, avec une dimension internationale non négligeable (plus d'un tiers des étudiants viennent d'une université à l'étranger).

\section{Les diplômés du Master... \\ Unsatisfied with the job I had landed after obtaining my Bachelor's Degree in Political science from a Canadian institution, I decided to return to University and complete a Master's Degree to climb the professional ladder. After searching for a while, I noticed that the offer of programs was too general for my needs and would not necessarily allow me to acquire the specific competences adapted to today's labor market.}

I expanded my search to Europe and came across an interesting academic opportunity at the University of Poitiers. A multi-disciplinary Master's degree focusing on the issues related to International Migrations, with the possibility to undertake professional or more research-oriented pathways seemed like the perfect fit for my needs.

I jumped on the opportunity and signed up for the program but was quite unsure about what was waiting for me. I was worried about the transition between academic cultures and not meeting the European standards.

The first few days in Poitiers dispelled my worries as I immediately felt welcomed. The proximity of the academic team with the students created a stimulating group dynamic. The teachings were of the utmost quality and covered a large diversity of topics related to the field of international migrations, through different academic lenses. Students are prepared within the first year of the program to complete a field study and to start producing scientific research through the completion of a Master's thesis. That first year was determining for me as it has completely re-oriented my professional aspirations. Writing those two Master's thesis was one of my greatest personal accomplishments, and I decided I would make it my career. I have started a PhD in Social and Political Geography at the University of Poitiers, still within the Migrinter research laboratory, where I am granted the freedom necessary to pursue my research and the support of a team of worldclass researchers who have treated me as one of their peers since day one. 
Martine BROUILLETTE, Doctorante au laboratoire Migrinter.

\begin{abstract}
l'UMR, ce qui permet d'inscrire dans la formation des collègues universitaires étrangers et des professionnels issus d'organisations internationales. Avec l'extension de ses réseaux de coopération scientifique, Migrinter a pu doter sa capacité de recherche d'une dimension européenne permettant d'inscrire la formation proposée dans une aire scientifique plus large, héritée notamment du réseau d'excellence Immigration Integration and Social Cohesion in Europe (IMISCOE). La formation a récemment signé un accord Erasmus avec l'Université de la Corogne en 2014. Elle projette d'élargir son projet d'internationalisation en déposant un dossier Erasmus Mundus impliquant d'autres partenaires étrangers qui constituent des collaborateurs scientifiques réguliers : en particulier en Afrique subsaharienne avec les Universités de Dakar et de Dschang.
\end{abstract}

\title{
L'ouverture internationale du Master au défi des pratiques pédagogiques
}

21 Dans le cadre d'un projet de coopération internationale en gestation, la formation s'appuie aussi bien sur les réseaux scientifiques constitués par les chercheurs que sur une circulation internationale étudiante déjà partiellement en place. De facto, le Master accueille dès la première année, via la procédure Campus France, de nombreux étudiants originaires des pays d'Afrique subsaharienne et plus spécifiquement de ceux d'Afrique de l'Ouest et du Centre. Cette attractivité internationale du Master repose sur la réputation d'enseignement de qualité dont jouit (à tort ou à raison) l'Université française dans les pays africains francophones. Elle s'explique aussi par la spécialisation du Master qui suscite l'intérêt du public étudiant africain sur les questions migratoires, en particulier quand elles sont associées à la problématique du développement des pays d'origine. Le rayon géographique de recrutement du master ne se limite pas à cette région puisque les étudiants viennent parfois de pays anglophones, d'Espagne, d'Italie ou d'Amérique Latine. Cela étant, par sa constance, le flux d'étudiants en provenance d'Afrique subsaharienne constitue certainement le plus important volume de diplômés venus de l'étranger. Une réflexion s'est alors ouverte sur l'idée de développer des formations axées sur les migrations internationales dans ces pays en particulier, tout en bénéficiant de l'expérience de l'UMR Migrinter en la matière.

À Dschang, au Cameroun, un programme de formation professionnelle spécialisé sur les migrations internationales, le Master professionnel "Migrations internationales, mondialisation et développement local » (MIMDEL), a vu le jour en 2010 avec l'appui technique et matériel de Migrinter. Cette initiative a permis, par le biais d'une convention de coopération universitaire, d'amorcer le comblement d'un vide dans le paysage académique national. Depuis 2008, à Dakar, l'Institut Population Développement et Santé de la Reproduction (IPDSR) a mis en place le Master «Migrations Internationales, Développement et Relations Interculturelles» (MIRI). L'enseignement a pour but de donner aux étudiants de différentes disciplines des SHS les fondamentaux pour appréhender à la fois les migrations internationales comme mouvement de population et comme fait de société. Les collaborations scientifiques et académiques passées et actuelles entre les chercheurs de l'Université de Dakar et de 
Migrinter constitueront sans doute, à l'avenir, un argument supplémentaire à l'élargissement au Sénégal d'un projet de formation internationale sur les migrations.

$\mathrm{Au}$ final, la formation en migrations internationales est confrontée, au niveau Master, à un triple défi: la pluridisciplinarité liée aux approches multiples de «l'objet migration »; la diversification des origines des étudiants et les débouchés offerts aux étudiants diplômés en " études migratoires ", menant de moins en moins vers le monde académique. Les enseignements dans leur forme et dans leur contenu doivent s'adapter à ces tendances. À ces trois premiers défis, notre expérience permet d'en ajouter un autre majeur : celui de la prise en compte des contextes nationaux dans la construction des formations et des approches pédagogiques.

Considérer les contextes nationaux permet de porter un regard critique sur la prévalence de certains angles d'enseignement des migrations internationales. Les différents environnements institutionnels, historiques et politiques du Sénégal, du Cameroun et de la France par exemple, se reflètent dans les formations et l'enseignement des migrations. En Afrique de l'Ouest et du Centre la question des migrations internationales a largement été abordée (besoin de connaissance et attente publique) sous l'angle de l'émigration internationale souvent qualifiée de " clandestine ", de sa mesure, de ses liens avec la pauvreté ; puis, cette question a été traitée par l'analyse des effets des circulations économiques sur le développement des pays d'origine en privilégiant la dimension « développementaliste » ou « utilitariste ». En revanche, en Europe, et en France en particulier, cette question a d'abord été traitée sous l'angle de l'immigration et de l'intégration, des relations interethniques et de plus en plus du transnationalisme. D'une certaine façon, les besoins en formation et la nature des enseignements semblent être ancrés dans ces représentations nationales ou régionales des phénomènes migratoires. Dès lors, deux questions se posent. Face à la diversité croissante des disciplines enseignées et/ou d'appartenance des enseignantschercheurs existe-t-il des concepts transversaux, voire un paradigme "support » en vue d'enseigner les migrations internationales dans une perspective pluridisciplinaire? Les échanges générés par l'idée d'un projet commun de formation axé sur les migrations et la mobilité des Hommes à l'échelle internationale peuvent-ils permettre de dépasser les paradigmes «mobilitaire», «développementaliste » et « transnational »?

Une première réponse à ces questions est apportée par Swanie Potot dans son intervention au colloque par une discussion critique du paradigme du transnationalisme. En effet, comme le "paradigme du transnationalisme » nous fait sortir des dichotomies pays d'émigration/pays d'immigration au risque de vulgariser à tout va le «transnational » et le «mondialisé », le "paradigme mobilitaire », qui semble s'installer à Migrinter et plus largement dans les études migratoires, (sur-)valorise peut-être "le mouvement» au risque de négliger les situations d'immobilité et ses significations sociales et politiques. Nous apportons ici un autre complément de réponse à partir de la diversité de traitement de la question migratoire selon les contextes de formation. Il existe une forte imbrication entre les contextes de production scientifique, les évolutions épistémologiques (construction du savoir sur les migrations) et les pratiques pédagogiques mises en œuvre (manière de transmettre les connaissances sur les migrations). Le projet d'internationalisation de la formation doit s'emparer de cette réflexion. 


\section{BIBLIOGRAPHIE}

Béteille, Roger (1981) Une nouvelle approche géographique des faits migratoires : champs, relations, espaces relationnels, L’Espace Géographique, vol. 10, n³ 3, pp. 187-197.

Chardonnel, Sonia ; Dureau, Françoise ; Kataba, Kamila ; Imbert, Christophe ; Levy, Jean-Pierre (2009) Les mobilités (agrégation 2010, Bibliographie), Historiens et Géographes, nº 407, pp. 232-241.

Clochard, Olivier (dir.) (2012) Atlas des migrants en Europe, Géographie critique des politiques migratoires, Paris, Armand Colin, $143 \mathrm{p}$.

Cortès, Geneviève ; Faret, Laurent (dir.) (2009) Les circulations transnationales. Lire les turbulences migratoires contemporaines, Paris, Armand Colin, 244 p.

Coulon, Alain (2007) L'école de Chicago, Paris, Presse universitaire de France, 127 p. (Que sais-je ?, $n^{\circ} 2639$ ).

Domenach, Hervé ; Picouet, Michel (1987) Le caractère de la réversibilité dans l'étude de la migration, Population, vol. $42, \mathrm{n}^{\circ}$ 3, pp. 469-483.

Doraï, Kamel (2006) Les réfugiés palestiniens du Liban. Une géographie de l'exil, Paris, CNRS Éditions, $252 \mathrm{p}$.

Dureau, Françoise (1999) Les mobilités à géométrie variable des habitants de Bogota, Espace Populations Sociétés, $\mathrm{n}^{\circ}$ 2, pp. 329-344 (La mobilité spatiale des populations).

Dureau, Françoise ; Hily, Marie-Antoinette (dir.) (2009) Les mondes de la mobilité, Rennes, Presse universitaire de Rennes, 189 p. (Essais).

Guengant, Jean-Pierre (1996) Migrations internationales et développement : les nouveaux paradigmes, Revue Européenne des Migrations Internationales, vol. 12, $\mathrm{n}^{\circ}$ 2, pp. 107-121.

Guillon, Michèle (1992) Étrangers et immigrés en Ile de France, Paris, Université de Paris 1,560 p. Th. État : Géogr. : Paris $1: 560$ p.

Hily, Marie-Antoinette (2009) L'usage de la notion de « circulation migratoire », in Cortès, G. ; Faret, L. (dir.) Les circulations transnationales, Paris, Armand Colin, pp. 23-28.

Hily, Marie-Antoinette ; Missaoui, Lamia (2002) Éditorial, Revue Européenne des Migrations Iinternationales, vol. 18, $\mathrm{n}^{\circ}$ 3, pp. 7-8 (L'étranger dans la ville).

Imbert, Christophe ; Dubucs, Hadrien ; Dureau, Françoise ; Giroud, Mathieu (2014) D'une métropole à l'autre. Pratiques urbaines et circulations dans l'espace européen, Paris, Armand Colin, 485 p. (Recherches).

Lacroix, Thomas ; Gonin, Patrick (2005) Migrations et développement : faux semblants et vrais enjeux, Géographes associés, $n^{\circ} 29$, pp. 89-101.

Lassailly-Jacob, Véronique (2003) Conditions d'accueil et insertion des réfugiés dans un pays d'Afrique Australe : la Zambie, in Guillon, M. ; Legoux, L. ; Ma Mung, E. (dir.) L'asile politique entre deux chaises. Droits de l'Homme et gestion des flux migratoires, Paris, L'Harmattan, pp. 245-265.

Ma Mung, Emmanuel ; Body-Gendrot, Sophie ; Hodeir, Catherine (1992) L'expansion du commerce ethnique : Asiatiques et Maghrébins dans la région parisienne, Revue Européenne des Migrations Internationnales, vol. 8, $\mathrm{n}^{\circ} 1$, pp. 39-59. 
Ma Mung, Emmanuel (dir.) (1992) Mobilités et investissements des émigrés. Maroc, Tunisie, Turquie, Sénégal, Paris, L'Harmattan, $272 \mathrm{p}$.

Ma Mung, Emmanuel ; Doraï, Kamel ; Hily, Marie-Antoinette ; Loyer, Frantz (1998) La circulation migratoire, bilan des travaux, Migrations études, ADRI (Agence pour le développement des relations interculturelles), pp. 1-12 [Disponible sur Internet]

Mauco, George (1932) Les migrations ouvrières en France au début du $19^{\text {ème }}$ siècle d'après les rapports des préfets de l'Empire de 1808 à 1813, Paris, Lesot, 74 p.

Noin, Daniel (1979) Géographie de la population, Paris, Masson, 310 p.

Robin, Nelly (1996) Atlas des migrations ouest-africaines vers l'Europe : 1985-1993, Paris, EurostatOrstom Éd., 110 p.

Simon, Gildas (1979) L'espace des travailleurs tunisiens en France. Structures et fonctionnement d'un champ migratoire international, Poitiers, Université de Poitiers, $426 \mathrm{p}$.

Th. Doct. : Géogr : 1979 : 426 p.

Simon, Gildas (1981) Réflexions sur la notion de champ migratoire international, Hommes et Terres $d u$ Nord, pp. 85-89 [Disponible sur Internet]

Simon, Gildas (2006) Migrations. La spatialisation du regard, Revue Européenne des Migrations Internationales, vol. $22, \mathrm{n}^{\circ} 2$, pp. 9-21.

Tarrius, Alain (1992) Les Fourmis d'Europe, Migrants riches, migrants pauvres et nouvelles villes internationales, Paris, L'Harmattan, 207 p.

Tarrius, Alain (2002) La mondialisation par le bas. Les nouveaux nomades de l'économie souterraine, Paris, Balland, $168 \mathrm{p}$.

\section{NOTES}

1. En témoigne la journée des doctorants qui a ouvert le colloque anniversaire des 30 ans de Migrinter et les différents rappels qui ont été faits par les directeurs successifs du laboratoire au cours de la table ronde introductive du colloque. On notera que parmi les directeurs, les trois derniers (William Berthomière, Kamel Doraï et Thomas Lacroix, directeur adjoint de Cédric Audebert) ont été formés à Migrinter dans le cadre du Master (ex-DEA) puis de l'école doctorale : un gage de pérennité dans la transmission de certains fondements théoriques de l'étude des migrations internationales et des valeurs pédagogiques dédiées à son enseignement.

2. Le colloque international «Penser les migrations pour repenser la société " s'est tenu à Poitiers du 21 au 24 juin 2016 (Migrinter CNRS/Université de Poitiers).

\section{INDEX}

Index géographique : France

Mots-clés : enseignement supérieur, méthodologie, coopération internationale 


\section{AUTEURS}

\section{DAVID LESSAULT}

Chargé de recherche en Géographie, Migrinter - UMR 7301, CNRS / Université de Poitiers david.lessault@univ-poitiers.fr

\section{ADELINA MIRANDA}

Professeure d'Anthropologie, Migrinter - UMR 7301, CNRS / Université de Poitiers adelina.miranda@univ-poitiers.fr

\section{VÉRONIQUE LASSAILLY-JACOB}

Professeure émérite de Géographie, Migrinter - UMR 7301, CNRS / Université de Poitier veronique.lassailly.jacob@univ-poitiers.fr 